\title{
Factores Determinantes para el Intraemprendimiento Social: El Caso de los Estudiantes de Ingeniería de la Universidad de La Serena, Chile
}

\author{
Segundo R. Cabana, Felicindo H. Cortés, Mauricio I. Aguilera y Felipe A. Vargas \\ Universidad de La Serena, Facultad de Ingeniería, Dpto. de Ing. Industrial, Casilla 554, La Serena-Chile \\ (e-mail: rcabana@userena.cl, fcortes@userena.cl, maguilera@userena.cl, fvargas@userena.cl)
}

Recibido Jul. 13, 2017; Aceptado Oct. 3, 2017; Versión final Nov. 16, 2017, Publicado Abr. 2018

\begin{abstract}
Resumen
Se presenta un modelo para determinar la conducta intraemprendedora social en estudiantes de Instituciones de Educación Superior, tomando como caso de estudio las carreras de Ingeniería de la Universidad de La Serena, en Chile. Se propone un modelo relacional con ocho hipótesis de trabajo. Se aplicaron encuestas a un grupo de 401 estudiantes, considerando escala politómicas tipo Likert. Para el contraste del modelo propuesto se emplearon los métodos de ecuaciones estructurales, basado en los mínimos cuadrados parciales. Se determinó que la innovación social, la fidelidad del estudiante y la disposición al riesgo explican en un $46,5 \%$ a la conducta intraemprendedora social. La identificación del estudiante con su carrera y con su universidad influye de forma directa y significativa en la fidelidad del estudiante. Se concluye que la gestión sistémica de la universidad se debe enfocar en promover la innovación social, fortaleciendo la disposición al riesgo de los estudiantes y mejorar los procesos de enseñanza aprendizaje e imagen institucional.
\end{abstract}

Palabras clave: intraemprendimiento social; innovación social; institución de educación superior; modelo de ecuaciones estructurales; fidelidad

\section{Determinant Factors for Social Intrapreneurism: The Case of the Engineering Students of the University of La Serena, Chile}

\begin{abstract}
A model to determine social intrapreneurism behavior in students of institutions of higher education is presented, taking as a study case the engineering careers of the University of La Serena, in Chile. A relational model with eight working hypotheses is proposed. A sample of 401 students was studied and a survey was applied, considering a Likert-type politomic scale. For comparing the results of the proposed model the method of structural equations, based on partial least squares was also used. It was determined that social innovation, student loyalty and willingness to risk account for $46.5 \%$ of social intra-entrepreneurial behavior, the identification of students with their career and their university influences directly and meaningfully student's fidelity. It is concluded that the systemic management of the university should focus on promoting social innovation, strengthening students' risk disposition and improving the processes of teaching learning and institutional image.
\end{abstract}

Keywords: social intrapreneurship; social innovation; institution of higher education; model of structural equations; fidelity 


\section{INTRODUCCIÓN}

En la actualidad la universidad es vista como una organización que tiene entre sus actividades la generación, difusión y transferencia de conocimiento, convirtiéndose en un actor fundamental de la nueva economía, sociedad y cultura, las que entienden al conocimiento como un factor estratégico que genera ventajas competitivas para permitir la diferenciación de las organizaciones y su sostenibilidad. Conscientes de esto, las universidades han empezado a dar mayor importancia a una de sus funciones sustantivas: la extensión universitaria o proyección social, mediante la cual esperan llevar conocimiento al entorno para contribuir al desarrollo local, regional, nacional e internacional. En este escenario los estudiantes universitarios tienen un rol fundamental al adquirir dichos conocimientos y comenzar a desarrollar su conducta intraemprendedora social, es decir, que gracias a sus actividades generen beneficios a nivel universitario y que repercutan positivamente en la sociedad.

Estudios recientes sobre los determinantes del logro académico de los universitarios destacan el apoyo social percibido. Su efecto deriva del sentimiento de mutua confianza hacia otras personas con las que se puede contar en caso de necesidad; esto mediatiza la valoración sobre las circunstancias problemáticas o estresantes y la apreciación de los recursos de que se dispone para afrontarlas (Martínez et al., 2014). Es por ello que las Instituciones de Educación Superior (IES), en particular la Universidad, como gran agente del cambio, son una factoría de conocimiento en muchos aspectos, puede con un modelo de intraemprendimiento Social organizado, influir en el tejido social próximo aportando y creando trabajo comunitario. Por lo tanto, la institución de educación como organización, sus líderes, profesores y alumnos; los entornos contextuales de la institución y la sociedad en general pueden salir beneficiados de los resultados de este trabajo (Lozano et al., 2015).

En Chile, actualmente las universidades se encuentran compitiendo por alumnos, recursos (humanos y de financiamiento) y reputación, siendo lo más importante los estudiantes. Este aumento en la competencia entre universidades es compartido en otros contextos. Así, en Europa, y en el marco del Espacio Europeo de Educación Superior, también se está produciendo un incremento en el nivel de competencia de las universidades en diversos aspectos (de la Fuente et al., 2010). Estos cambios importantes, junto a un aumento de las expectativas de la sociedad en lo que concierne a la labor de universidades estatales y las demandas de los usuarios de estos servicios, han causado grandes problemas y una preocupación por mejorar la calidad de enseñanza, investigación y todos los servicios que una universidad proporciona. Este interés ha conducido a dar énfasis mayor sobre el análisis de dos aspectos que se encuentran estrechamente ligados: calidad y satisfacción (de la Fuente et al., 2010). Por ello, el proceso educativo, es una actividad crucial en toda universidad y puede ser caracterizado en forma simple como un proceso interactivo e intencionado; interactivo, no solo por la existencia de relaciones humanas entre sus miembros sino también por la interacción que se da con una multiplicidad de factores asociados a este proceso (Bertoglia, 2005)

Por otra parte, cuando existe una correcta relación entre la Universidad y sus estudiantes, y estos tienen la convicción de generar nuevas ideas que nazcan de una necesidad de la misma universidad, se realizara un proyecto desarrollado por medio de estudiantes con conductas intraemprendedoras. Generándose actividades desde dentro de la universidad, que son beneficiosas no solo para su casa de estudios, sino que también puede generar impactos favorables para la sociedad, y entrenar a un futuro intraemprendedor de carácter corporativo y/o emprendedor que tenga sus propias ambiciones, que genere impacto en las empresas, la comunidad y el mundo (Salcedo, 2012).

En este contexto el estudio que se presenta se basa en ocho hipótesis relacionadas con los conceptos de satisfacción, identificación, fidelidad, innovación, disposición al riesgo y conducta intraemprendedora social presente en los estudiantes de la Facultad de Ingeniería de la Universidad de La Serena. Hipótesis 1 (H1): la satisfacción proceso enseñanza aprendizaje influye directa y positivamente sobre la identificación del estudiante con la Universidad; Hipótesis 2 (H2): la satisfacción imagen institucional influye directa y positivamente sobre la identificación del estudiante con la Universidad; Hipótesis $3(\mathrm{H} 3)$ : la satisfacción proceso apoyo interno influye directa y positivamente sobre la identificación del estudiante con la Universidad; Hipótesis $4(\mathrm{H} 4)$ : la satisfacción proceso apoyo social profesional influye directa y positivamente en la identificación del estudiante con la Universidad; Hipótesis 5 (H5): la identificación del estudiante con su Universidad influye directa y positivamente sobre la fidelidad del estudiante con su Universidad; Hipótesis 6 (H6): la fidelidad del estudiante con su Universidad influye directa y positivamente en la conducta intraemprendedora social; Hipótesis 7 (H7): la innovación social influye directa y positivamente en la conducta intraemprendedora social; e Hipótesis 8 (H8): la disposición al riesgo influye directa y positivamente en la conducta intraemprendedora social. 


\section{ANTECEDENTES}

Estudios anteriores han encontrado relación estadística entre las hipótesis mencionadas; Kumar y Jauhari (2016) evidenciaron que la satisfacción de los procesos de enseñanza aprendizaje y el desempeño influyen en la identificación de los miembros de una organización, logrando representar más del $45 \%$ de la varianza explicada, del mismo modo Annamdevula y Bellamkonda (2016), en una investigación realizada a tres de las universidades más antiguas de la India, evidencian que la satisfacción del estudiante puede ser determinada a partir de la calidad de enseñanza entregada por sus docentes, lo que es consistente con lo expresado por de la Fuente et al. (2010). Por otra parte, Sung y Yang (2008) comprueban que el impacto de la imagen institucional y los constructos relacionados con la imagen en la actitud de apoyo de los estudiantes representa el compromiso del estudiante con su organización y la identificación con ella. Con respecto a la innovación social y al intraemprendimiento social investigaciones evidencian la relación entre ambas variables, es así como Esen y Sekerdil (2017), aseguran que la innovación social es determinante del intraemprendimiento social, lo que está en línea con lo expuesto por Moriano et al. (2014) y Moriano et al. (2009). Dado las investigaciones anteriores se propone el modelo causal presentado en la figura 1, el que se sustenta en las siguientes relaciones.

\section{Identificación del estudiante y satisfacción proceso enseñanza aprendizaje}

La satisfacción del proceso enseñanza aprendizaje, es la apreciación favorable que hacen los estudiantes de los resultados y experiencias asociadas con su educación, en función de la atención a sus propias necesidades y al logro de sus expectativas (Candela et al., 2013). Existen diversas investigaciones que estudiaron los efectos positivos de la confianza y apoyo de los profesores. Cokley et al. (2004) encontraron que la gran mayoría de los estudiantes consideraron importante la relación con sus docentes para su desarrollo. Willie (2000) señala que a través de relaciones interpersonales de respeto y confianza se pueden transformar los ambientes de aprendizaje en verdaderas comunidades de aprendizaje. McNeely y Falci (2004) encontraron que los adolescentes que perciben apoyo de sus profesores se comprometen más con su institución de educación y son menos vulnerables a involucrarse en conductas de riesgos. Klem y Connell (2004), reconociendo que el vínculo con las instituciones de educación es importante para el aprendizaje y que los estudiantes a través de los años de estudio viven una progresiva desvinculación con estos, en su investigación encontraron que el apoyo de los profesores facilitaba mantener los lazos con sus escuelas. En la misma línea, Yáñez et al. (2005) comprobaron que la confianza hacia los docentes era un determinante significativo en la actitud de los estudiantes para creer en el valor de la evaluación del desempeño docente y en la disposición positiva hacia responderla.

\section{Identificación del estudiante y satisfacción imagen institucional}

Diversas investigaciones confirman que la forma en cómo es percibida la organización (la evaluación que se haga de ella, su imagen) va a influir en la identificación. Asimismo, diversas investigaciones han explorado la influencia sobre la identificación de diferentes características de la identidad de la organización que favorecen la satisfacción de dichas necesidades de autodefinición; en esta línea, se ha demostrado que el prestigio o reputación de la organización mejora el atractivo de la identidad y la identificación. De esta forma, la imagen de marca de la universidad se fundamentará en bases sólidas y relevantes para sus egresados, influyendo directamente en sus niveles de identificación para con la institución, siendo capaces de defenderla y sentirse parte de ella, así como en los niveles de lealtad, con lo cual aumentará la intención de elegirla de nuevo para estudios de postgrado, seguir en contacto con ella, recomendarla o hablar bien de ella en su entorno (Scott y Lane, 2000).

También contribuyen a la formación de la imagen, y de manera decisiva, las informaciones provenientes del entorno de la organización. Esta última afirmación debe ser destacada, puesto que no sólo los mensajes enviados por la organización influyen o afectan a la formación de la imagen, sino también todos aquéllos que puedan provenir de otras fuentes y que contengan información sobre la organización o sus actividades, como también aquéllos que hagan referencia a su ámbito de trabajo o acción (sector al que pertenezca), que puedan afectar indirectamente su imagen (Capriotti, 2013).

\section{Identificación del estudiante y satisfacción proceso apoyo interno}

La comunicación corporativa, sirve para recordar a los miembros su afiliación actual y generar una consideración positiva de su identidad organizativa (Scott y Lane, 2000). Una herramienta útil de comunicación son las webs corporativas, donde se facilita a los clientes chatear, información sobre acciones de patrocinio, blogs, clubs, foros o chats. La frecuencia y calidad de comunicación de la universidad en las relaciones con el alumno, incrementa la relevancia que para el alumno tiene la universidad, convirtiéndolo psicológicamente en miembro legítimo de la misma. Es una acción que favorece la identificación por los 
grandes beneficios que tiene para una empresa el estado ideal de relaciones con el cliente, en la medida en que refuerza la lealtad del consumidor y activa comportamientos extraordinarios de apoyo a la empresa (Pratt, 1998). Moriano et al. (2009) confirmaron a través de un estudio la importancia de la identificación organizacional como variable psicosocial mediadora del impacto de los factores de la organización sobre el intraemprendimiento social de sus miembros. Donde el factor organizacional "apoyo de la dirección" que forma parte de la satisfacción del proceso apoyo interno, no muestra un impacto significativo directo sobre el intraemprendimiento social, pero si influye significativamente a través de la identidad organizacional.

\section{Identificación del estudiante y satisfacción proceso apoyo social profesional}

Desde el enfoque de la identidad social aplicado al trabajo se viene insistiendo en que la identidad social es la base para recibir apoyo social dentro de un grupo y de una organización. De hecho, hay tres formas distintas en las cuales la identificación puede afectar al apoyo social: por la disponibilidad de un miembro del grupo a brindar apoyo social a otro, por la probabilidad de que la persona necesitada de apoyo, lo acepte y finalmente, por la interpretación que ésta haga del ofrecimiento de apoyo que recibe. Como han demostrado estos estudios, dar apoyo social es contingente con las percepciones de una identidad social compartida, en donde, es más probable que una persona brinde apoyo social a un miembro de su propio grupo, es más probable que el apoyo social sea aceptado cuando quien lo brinda es percibido como miembro de una categoría social compartida y es más probable que las intenciones con las que se ofrece apoyo sean mejor interpretadas cuando ambas personas pertenecen al mismo grupo social (Topa et al., 2010). De esta forma la satisfacción del estudiante en su inserción en la universidad, contribuye a reafirmar su compromiso institucional (Donoso y Schiefelbein, 2007).

\section{Fidelidad e identificación del estudiante}

Un estudio realizado por Schlesinger et al. (2014), confirma la influencia de la Identificación sobre la fidelidad y el compromiso. Dada las características actuales del entorno de las universidades, tales como la reducción del número de estudiantes que ingresan y el aumento de los que abandonan los estudios, entre otros, justifican la importancia de analizar la fidelidad en este ámbito, la cual es necesaria para la supervivencia de las instituciones de enseñanza superior (Helgesen y Nesset, 2007). Después de egresado, un alumno identificado con la institución puede atraer nuevos alumnos a través de una comunicación boca-oreja positiva, mejorar la imagen y reputación de la universidad en su entorno o atraer entes $u$ organizaciones que hagan donaciones o financien proyectos de investigación. De esta forma, se puede ver cómo las consecuencias de esta identificación, generan fidelidad, la cual no está limitada al tiempo que permanece el alumno en la institución, sino que continúan a lo largo de su vida (Fullerton, 2011). Las estrategias centradas en mejorar la satisfacción del estudiante y su fidelidad se deben convertir en decisiones estratégicas en las IES, instalando un paradigma de gestión educacional prioritario en Chile, donde la calidad de la educación es simultáneamente una meta de corto plazo como también un objetivo estratégico, que debe impactar en las decisiones de los gestores educacionales (Cabana et al., 2016).

\section{Intraemprendimiento social en instituciones de educación y fidelidad del estudiante}

En la educación superior, la fidelidad del usuario se plasmaría tanto en su disposición a retornar a la misma para seguir formándose como en la posibilidad de recomendar la universidad a otros potenciales usuarios pertenecientes a su entorno (trabajo, familia, vecindad), generando así actitudes positivas hacia la institución (Pereira, 2011). Para la universidad, en su papel educativo, no sólo debe formar al estudiante para enfrentarse al dinámico mercado de trabajo que requiere respuestas rápidas, innovadoras y especializadas, sino que también debe contribuir en su formación ciudadana, durante este proceso de formación de los estudiantes, la universidad debería inculcar el sentido de agradecimiento por la institución y de retribución por su aporte en su desarrollo formativo, generando un vínculo emocional de fidelidad (Petrella, 2008).

\section{Intraemprendimiento social en instituciones de educación e innovación social}

El intraemprendimiento social trata sobre la posibilidad de que los empleados tomen su valor en el lugar de trabajo y se atrevan a hacer algo con ello, su materialidad se traducirá en un comportamiento basado en la motivación intrínseca y que da como resultado altos niveles de compromiso. La teoría sugiere que cuando las personas se dedican, da lugar a una mayor creatividad y mayor compromiso para hacer que sus iniciativas sean un éxito. Eso es lo que se necesita para que las empresas sean más sostenibles y crear paso a paso un impacto más positivo en el mundo (Nijhof et al., 2012).

El intraemprendimiento social, no sólo localiza las mejores mentes para encontrar ideas y soluciones, sino que implica a estas personas en el proceso de innovación, dejándolas convertir sus ideas en proyectos y ver como estos proyectos derivan en nuevos negocios. La innovación se refiere al compromiso de la institución 
para crear e introducir nuevos productos, servicios y procesos (Romero, 2012). Por lo tanto, la innovación social se asienta como un marco conceptual eficaz del hacer universitario desde la responsabilidad social (moral) que le compete, en organización integrada, en un mundo de vertiginoso cambio y creciente complejidad. Es una manera de entender el sentido social de la actividad universitaria en aras al desarrollo personal, cívico-social, político y cultural de las personas, las comunidades y los pueblos. Está vinculada con la propia función universitaria, enraizada en su misión y visión (Villa, 2014).

\section{Intraemprendimiento social en instituciones de educación y disposición al riesgo}

La disposición al riesgo puede definirse como la preferencia por las situaciones que pueden reportar beneficiosas recompensas en caso de éxito, pero también severas consecuencias si el individuo fracasa. De esta forma, el intraemprendedor se aventura dentro de áreas desconocidas para la organización, sin saber cuáles serán los resultados. Se refiere a la disposición del sujeto a comprometerse con fuentes de oportunidades bajo posibilidades de fracaso (Moriano et al., 2009). Los factores personales son una parte importante al momento de realizar un perfil emprendedor, ya que se está hablando directamente del individuo. Los investigadores han tratado de poner en relieve los aspectos psicológicos y características personales del emprendedor. El emprendedor debe ser flexible e imaginativo; debe asumir riesgos con el convencimiento de que puede fracasar, y buscar segundas oportunidades de los fracasos, ya que la experiencia que se obtiene le ofrece más seguridad para iniciar un nuevo proyecto. Estas situaciones pueden ser no deseadas ni esperadas; de esta manera se hace necesario que la persona joven, que tiene menos experiencia, conozca las pautas para evitar caer en los errores más usuales. Así, desde diferentes teorías se enfatiza la necesidad de realización o la propensión a la toma de riesgo como factores que son determinantes del espíritu emprendedor (Quintero, 2007).

\section{Modelo causal}

Considerando todas las relaciones anteriores, se propone un modelo causal que busca identificar las variables que influyen en la conducta intraemprendedora social en estudiantes de ingeniería de Instituciones de Educación Superior, con foco en Universidad de La Serena (Fig. 1).

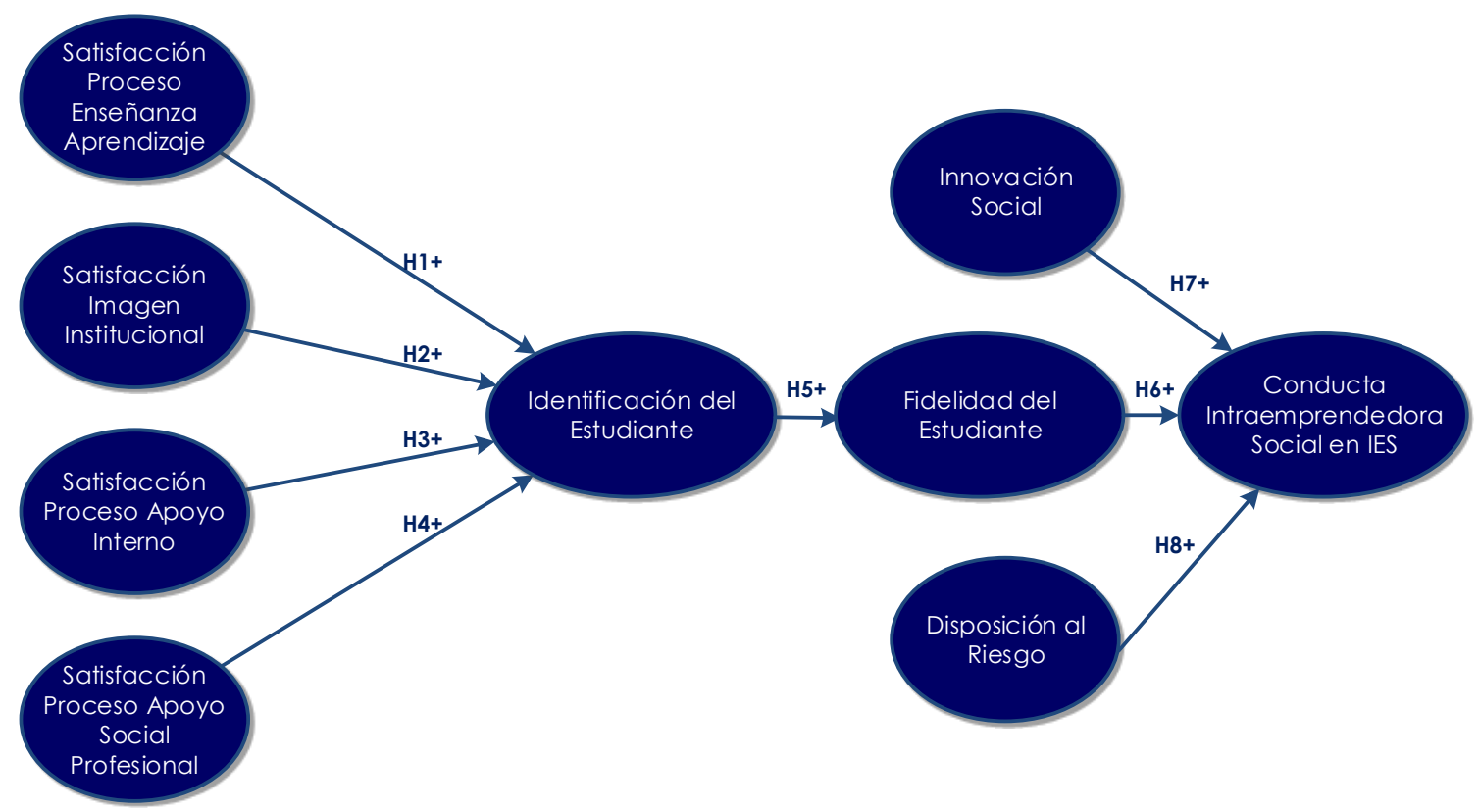

Fig. 1: Modelo causal sobre la Conducta Intraemprendedora Social en Instituciones de Educación Superior

\section{METODOLOGIA}

Para validar el modelo propuesto, se presenta un estudio empírico, donde el método empleado para recoger la información es de carácter cuantitativo basado en una encuesta estructurada de 57 preguntas de tipo Likert, escala de 1 (mínimo valor asociado a respuesta) a 5 (máximo valor asociado a respuesta), el tipo de muestreo es probabilístico con un nivel de confianza del 95\%. La encuesta abarcó los siguientes ítems, satisfacción, entre los cuales se diferencia según su procedencia, (Proceso apoyo social, proceso enseñanza aprendizaje, proceso de apoyo interno, e imagen institucional), identificación de estudiante con su carrera, fidelización del alumno con su universidad, innovación, disposición a asumir riesgos, y conducta intraemprendedora social en IES, el cual fue evaluado a partir de las siguientes dos preguntas ¿Ejecuto acciones sociales o 
intraemprendimientos sociales que logran generar valor social en la Universidad/carrera o actividades internas? ¿Ejecuto acciones sociales o intraemprendimientos sociales que logran generar valor social en la sociedad? El trabajo de campo se realizó de manera personal y vía online, entre diciembre del año 2015 y mayo del año 2016, se eliminaron datos que presentaban errores de medida e incongruencias, representadas con una desviación típica de 0 . Los casos válidos ascendieron a 401, siendo todos estudiantes de la Facultad de Ingeniería de la Universidad de La Serena (ver tabla 1).

Tabla 1: Ficha técnica de la investigación

\begin{tabular}{|l|l|}
\hline Tipo de muestreo & Muestreo probabilístico \\
\hline Nivel de confianza & $95 \% ; \mathrm{z}=1,96 ; \mathrm{p}=\mathrm{q}=0,5$ (5\% error y 95\% de confiabilidad) \\
\hline Tamaño muestral & 401 estudiantes (población de 2934 estudiantes) \\
\hline Unidad de análisis & Facultad de Ingeniería de la Universidad de La Serena \\
\hline Método de recogida de la información & Cuestionario presencial y online de 57 preguntas \\
\hline Tipo de pregunta encuesta & $\begin{array}{l}\text { Politómicas tipo Likert (1 a 5) puntuaciones o categorías y de } \\
\text { selección }\end{array}$ \\
\hline Fecha realización del trabajo de campo & Entre diciembre de 2015 y mayo de 2016 \\
\hline
\end{tabular}

\section{Análisis Descriptivo}

Los resultados revelan que la muestra se integra por $70,73 \%$ de hombres y $29,27 \%$ de mujeres, provenientes en su mayoría de establecimientos educacionales de carácter particular subvencionado (77,99\%). El ingreso familiar mensual más habitual es desde los US\$ 300 a los US\$ 899,9, representando el $45,43 \%$ de los casos. En tanto la carrera con mayor cantidad de encuestados es la de Ingeniería Civil Industrial con un 13,35\% de la muestra y el ciclo educacional con mayor participación es el segundo, correspondiente a estudiantes que cursan los niveles desde el quinto al octavo representando 58,13\% del total de estudiantes (Ver Tabla 2).

Tabla 2: Datos descriptivos de la muestra. Características socio-demográficas de la muestra

\begin{tabular}{|c|c|c|c|c|c|}
\hline Variable & Ítem & $\begin{array}{c}\text { Porcentaje } \\
\text { de la Muestra }\end{array}$ & Variable & Ítem & $\begin{array}{l}\text { Porcentaje en } \\
\text { la Muestra }\end{array}$ \\
\hline \multirow{2}{*}{ Género } & Hombre & $70,73 \%$ & \multirow{3}{*}{ Ciclo } & Ciclo 1 & $18,93 \%$ \\
\hline & Mujer & $29,27 \%$ & & Ciclo 2 & $58,13 \%$ \\
\hline \multirow{10}{*}{ Carrera } & Arquitectura & $11,94 \%$ & & Ciclo 3 & $22,93 \%$ \\
\hline & Ing. Civil & $8,67 \%$ & \multirow{4}{*}{ Establecimiento Escolar } & Municipal & $16,63 \%$ \\
\hline & $\begin{array}{l}\text { Ing. Civil } \\
\text { Ambiental }\end{array}$ & $5,85 \%$ & & $\begin{array}{l}\text { Particular } \\
\text { Subvencionado }\end{array}$ & $77,99 \%$ \\
\hline & $\begin{array}{l}\text { Ing. Civil de } \\
\text { Minas }\end{array}$ & $13,82 \%$ & & \multirow{2}{*}{ Particular } & \multirow{2}{*}{$5,39 \%$} \\
\hline & $\begin{array}{l}\text { Ing. Civil } \\
\text { Industrial }\end{array}$ & $13,35 \%$ & & & \\
\hline & $\begin{array}{l}\text { Ing. Civil } \\
\text { Mecánica }\end{array}$ & $9,84 \%$ & \multirow{5}{*}{$\begin{array}{l}\text { Ingreso Familiar Total } \\
\text { (Bruto) }\end{array}$} & US\$ 0 - US\$299,9 & $8,67 \%$ \\
\hline & $\begin{array}{l}\text { Ing. de Ejecución } \\
\text { en Minas }\end{array}$ & $12,41 \%$ & & US\$ 300 - US\$ 899,9 & $45,43 \%$ \\
\hline & $\begin{array}{l}\text { Ing. de Ejecución } \\
\text { Mecánica }\end{array}$ & $9,84 \%$ & & US\$ 900 - US\$1.799,9 & $26,70 \%$ \\
\hline & $\begin{array}{l}\text { Ing. en } \\
\text { Alimentos }\end{array}$ & $3,75 \%$ & & $\begin{array}{l}\text { US } \$ 1.800-\text { US } \$ \\
2.999,9\end{array}$ & $11,94 \%$ \\
\hline & $\begin{array}{l}\text { Ing. en } \\
\text { Construcción }\end{array}$ & $10,54 \%$ & & más de US\$3000 & $7,26 \%$ \\
\hline
\end{tabular}

Fiabilidad individual de los indicadores

Con la finalidad de evaluar la viabilidad del análisis factorial, se cuantificó el índice Kaiser-Meyer-Olkin (KMO) que para cada uno de los factores fue superior a 0,5 y la prueba de esfericidad de Bartlett (PEB) la que debe ser significativa $(p<0,05)$, (Méndez y Rondón, 2012). Para esto se utilizó el software SmartPLS en su versión 3.2.6 utilizando el método de extracción Bootstrap. Al respecto, para aceptar un indicador como integrante de un constructo se consideró las cargas factoriales con valores superiores a 0,4 sugerido por Hair et al. (2009), 
sin embargo, para obtener más rigurosidad en los datos se eliminaron aquellas cargas factoriales con valores inferiores a 0,5. La tabla 3 denota que los indicadores FEU021, SPEA043, SPEA044, SPEA048, SPEA049, SIGI051, SPASP071, SPASP072, IS081, IS082 y DAR094 fueron eliminadas por no cumplir con el mínimo establecido.

Tabla 3: Cargas Factoriales de los Indicadores

\begin{tabular}{|c|c|c|}
\hline Constructo & Indicador & Carga Factorial \\
\hline \multirow[t]{5}{*}{ Fidelidad del Estudiante con su Carrera y Universidad (FEU) } & FEU021 & 0,482 \\
\hline & FEU022 & 0,722 \\
\hline & FEU023 & 0,874 \\
\hline & FEU024 & 0,605 \\
\hline & FEU025 & 0,844 \\
\hline \multirow[t]{5}{*}{ Identificación del Estudiante con su Carrera y Universidad (IEC) } & IEC031 & 0,798 \\
\hline & IEC032 & 0,845 \\
\hline & IEC033 & 0,858 \\
\hline & IEC034 & 0,650 \\
\hline & IEC035 & 0,688 \\
\hline \multirow[t]{10}{*}{ Satisfacción del Proceso Enseñanza Aprendizaje (SPEA) } & SPEA041 & 0,728 \\
\hline & SPEA042 & 0,695 \\
\hline & SPEA043 & 0,379 \\
\hline & SPEA044 & 0,301 \\
\hline & SPEA045 & 0,714 \\
\hline & SPEA046 & 0,771 \\
\hline & SPEA047 & 0,684 \\
\hline & SPEA048 & 0,055 \\
\hline & SPEA049 & 0,168 \\
\hline & SPEA050 & 0,661 \\
\hline \multirow[t]{6}{*}{ Satisfacción Imagen Institucional (SIGI) } & SIGI051 & 0,464 \\
\hline & SIGI052 & 0,629 \\
\hline & SIGI053 & 0,778 \\
\hline & SIGI054 & 0,765 \\
\hline & SIGI055 & 0,727 \\
\hline & SIGI056 & 0,811 \\
\hline \multirow[t]{5}{*}{ Satisfacción Procesos de Apoyo Interno (SPAI) } & SPAI061 & 0,654 \\
\hline & SPAI062 & 0,771 \\
\hline & SPAI063 & 0,730 \\
\hline & SPAI064 & 0,663 \\
\hline & SPAI065 & 0,719 \\
\hline \multirow[t]{4}{*}{ Satisfacción Proceso Apoyo Social Profesional (SPASP) } & SPASP071 & 0,487 \\
\hline & SPASP072 & 0,314 \\
\hline & SPASP073 & 0,882 \\
\hline & SPASP074 & 0,892 \\
\hline \multirow[t]{5}{*}{ Innovación Social (ISO) } & IS081 & 0,203 \\
\hline & IS082 & 0,086 \\
\hline & IS083 & 0,742 \\
\hline & IS084 & 0,812 \\
\hline & IS085 & 0,788 \\
\hline \multirow[t]{4}{*}{ Disposición al Riesgo (DAR) } & DAR091 & 0,837 \\
\hline & DAR092 & 0,864 \\
\hline & DAR093 & 0,815 \\
\hline & DAR094 & 0,369 \\
\hline \multirow[t]{2}{*}{ Conducta Intraemprendedora Social en IES (CIIES) } & CIIES010 & 0,906 \\
\hline & CIIES011 & 0,912 \\
\hline
\end{tabular}




\section{Fiabilidad y Validez del constructo}

Para evaluar la fiabilidad del constructo se debe tener en cuenta la consistencia interna, la que exige que el Alpha de Cronbach sea mayor a 0,7 (Forcael et al., 2013). El alfa de Cronbach para cada factor por separado no tiene en cuenta la influencia sobre la fiabilidad del resto de constructos. Por esta razón, se realiza el cálculo de la fiabilidad compuesta (IFC) la cual sí tiene en cuenta las interrelaciones de los constructos, además tiene como valor mínimo 0,7. En tabla 4 se observa que la fiabilidad compuesta (IFC) y a de Cronbach presentan una alta consistencia interna en todos los constructos analizados, ambas medidas superan los niveles recomendados de 0,7 . Se verifica por tanto la consistencia interna de las variables del instrumento de evaluación.

Para valorar la validez del constructo se realizan dos análisis fundamentales: la validez convergente y la validez discriminante. La validez convergente se comprueba a través de la varianza extraída media (AVE) de los constructos, de tabla 4, el coeficiente AVE para todos los valores alcanzados superan el valor mínimo recomendado de 0,5 y son significativos al $95 \%$, por lo que se puede afirmar que las variables explican más del $50 \%$ de la varianza de sus constructos y por lo tanto existe validez convergente (Esposito et al., 2010). La validez discriminante expuesta en la tabla 5 , indica en qué medida un constructo es diferente a los otros constructos que conforman el modelo. Una forma de comprobar este criterio de validación, es demostrar que las correlaciones entre los constructos son más bajas que la raíz cuadrada de la varianza extraída media AVE (Forcael et al., 2013). Los valores alcanzados, verifican que los constructos presentan adecuados índices de fiabilidad y validez.

Tabla 4: Fiabilidad y Validez Convergente de los Constructos

\begin{tabular}{|l|c|c|c|}
\hline \multicolumn{1}{|c|}{ Constructo } & $\begin{array}{c}\text { Alfa de } \\
\text { Cronbach }\end{array}$ & $\begin{array}{c}\text { Índice de } \\
\text { Fiabilidad } \\
\text { Compuesta (IFC) }\end{array}$ & $\begin{array}{c}\text { Validez } \\
\text { Convergente } \\
\text { (AVE) }\end{array}$ \\
\hline Satisfacción del Proceso Enseñanza Aprendizaje (SPEA) & 0,807 & 0,859 & 0,597 \\
\hline Satisfacción Imagen Institucional (SIGI) & 0,802 & 0,861 & 0,554 \\
\hline Satisfacción Proceso de Apoyo Social Profesional (SPASP) & 0,730 & 0,881 & 0,787 \\
\hline Satisfacción Proceso de Apoyo Interno (SPAI) & 0,752 & 0,834 & 0,502 \\
\hline Identificación del Estudiante con su Carrera y Universidad (IEC) & 0,830 & 0,880 & 0,597 \\
\hline Fidelidad del Estudiante con su Carrera y Universidad (FEU) & 0,765 & 0,850 & 0,591 \\
\hline Innovación Social (ISO) & 0,702 & 0,833 & 0,625 \\
\hline Disposición al Riesgo (DAR) & 0,792 & 0,877 & 0,704 \\
\hline Conducta Intraemprendedora Social en IES (CIIES) & 0,790 & 0,905 & 0,826 \\
\hline
\end{tabular}

Tabla 5: Validez Discriminante de los Constructos

\begin{tabular}{|l|c|c|c|c|c|c|c|c|c|}
\cline { 2 - 11 } \multicolumn{1}{c|}{} & CIIES & DAR & FEU & IEC & ISO & SPEA & SIGI & SPASP & SPAI \\
\hline CIIES & 0,909 & & & & & & & & \\
\hline DAR & 0,466 & 0,839 & & & & & & & \\
\hline FEU & 0,139 & 0,120 & 0,769 & & & & & & \\
\hline IEC & 0,115 & 0,142 & 0,529 & 0,772 & & & & & \\
\hline ISO & 0,674 & 0,573 & 0,147 & 0,163 & 0,790 & & & & \\
\hline SPEA & 0,113 & 0,108 & 0,610 & 0,431 & 0,128 & 0,710 & & & \\
\hline SIGI & 0,139 & 0,065 & 0,460 & 0,348 & 0,133 & 0,470 & 0,745 & & \\
\hline SPASP & 0,224 & 0,226 & 0,116 & 0,159 & 0,295 & 0,221 & 0,172 & 0,887 & \\
\hline SPAI & 0,069 & 0,017 & 0,278 & 0,230 & 0,048 & 0,373 & 0,500 & 0,188 & 0,709 \\
\hline
\end{tabular}

\section{RESULTADOS}

Se planteó un análisis de ecuaciones estructurales para el contraste del modelo de relaciones propuesto (Fig. 1), que se desarrolló mediante el software SmartPLS, del cual se obtuvo también la varianza explicada y la validez predictiva del modelo.

\section{Coeficiente de determinación y validez predictiva del modelo}

El coeficiente de determinación de las variables endógenas o dependientes $\left(R^{2}\right)$ debe ser igual o mayor que 0,1 como valor mínimo (Falk y Miller, 1992). Por otra parte, lo que determina la calidad de predicción del 
modelo estructural es el Test de Stone-Geisser $\left(\mathrm{Q}^{2}\right)$. Este test se usa como criterio para medir la relevancia predictiva de los constructos dependientes y se calcula por medio de la técnica Blindfolding. En el caso de que la $\mathrm{Q}^{2}>0$, indica que el modelo tiene relevancia predictiva (Chin, 1998). En la tabla 6, se puede apreciar como el valor $\mathrm{R}^{2}$ para la Conducta Intraemprendedora Social es de 0,465 que quiere decir que el 46,5\% de la varianza de este constructo es explicada de forma satisfactoria. A partir de este criterio empírico todos los constructos poseen una calidad de poder de predicción aceptable y en todos ellos los valores de $\mathrm{Q}^{2}$ son positivos, lo cual certifica la relevancia predictiva del modelo.

Tabla 6: Varianza explicada y validez predictiva del modelo

\begin{tabular}{|c|c|c|}
\hline Constructo & $R^{2}$ & $Q^{2}$ \\
\hline Conducta Intraemprendedora Social en IES & 0,465 & 0,364 \\
\hline Fidelidad del Estudiante con su Universidad & 0,280 & 0,154 \\
\hline Identificación del Estudiante con su Carrera & 0,216 & 0,115 \\
\hline
\end{tabular}

\section{Bondad de ajuste y contraste de hipótesis}

Esposito et al. (2010) sugieren un criterio global de bondad de ajuste para los modelos estructurales PLS, proponen que el índice de bondad de ajuste global se dé por medio de la raíz cuadrada de la multiplicación de la media aritmética del análisis de la varianza extraída (AVE) y la media aritmética del coeficiente de determinación $\left(\mathrm{R}^{2}\right)$ de las variables endógenas o dependientes. Como se observa en tabla 7, el índice de bondad de ajuste (GoF) del modelo de análisis es de 0,454 demostrando que se tiene un buen ajuste en el modelo de medida y en el modelo estructural, cumpliendo así con el criterio empírico de que la medida de bondad de ajuste debe variar entre 0 y 1, a mayor valor, mejor será el índice (Tenenhaus et al., 2005).

\section{Análisis de relaciones causales y contraste de hipótesis}

La metodología PLS no presume que la información está normalmente distribuida, lo que significa que, para evaluar la calidad del modelo completo, es necesario aplicar una técnica de re muestreo no paramétrico llamado bootstrapping, la que involucra re muestreo aleatorio con reposición de la muestra original, creando nuevas pseudo-muestras a partir de la muestra original con el objetivo de obtener los errores muestrales para testeo de hipótesis. La nueva muestra obtenida por este proceso permite la estimación de coeficientes en la metodología PLS-SEM para testear su significancia estadística (Hair et al., 2011). Esta técnica, ofrece el cálculo del error estándar de los parámetros, donde se debe verificar la condición del estadístico T>= 1,96 para poder determinar su nivel de significancia (ver tabla 7) (Kwong, 2013).

Tabla 7: Modelo de Ecuaciones Estructurales: Análisis de relaciones causales y contraste de hipótesis.

\begin{tabular}{|c|l|c|c|c|c|}
\hline Hipótesis & \multicolumn{1}{|c|}{ Relaciones } & Coeficiente PATH & Estadístico $T$ & $P$ Value & Contraste \\
\hline H1 & SPEA $\rightarrow$ IEC & 0,333 & 6,750 & 0,000 & ACEPTA \\
\hline H2 & SIGI $\rightarrow$ IEC & 0,179 & 3,550 & 0,000 & ACEPTA \\
\hline H3 & SPAI $\rightarrow$ IEC & 0,006 & 0,104 & 0,917 & RECHAZA \\
\hline H4 & SPASP $\rightarrow$ IEC & 0,054 & 1,181 & 0,238 & RECHAZA \\
\hline H5 & IEC $\rightarrow$ FEU & 0,529 & 13,061 & 0,000 & ACEPTA \\
\hline H6 & FEU $\rightarrow$ CIIES & 0,101 & 2,248 & 0,047 & ACEPTA \\
\hline H7 & ISO $\rightarrow$ CIIES & 0,602 & 16,045 & 0,000 & ACEPTA \\
\hline H8 & DAR $\rightarrow$ CIIES & 0,117 & 2,427 & 0,016 & ACEPTA \\
\hline Índice de Bondad de Ajuste (GoF): 0,454 & & \\
\hline
\end{tabular}

\section{DISCUSIÓN}

Los resultados alcanzados establecen que la innovación social, la fidelidad del estudiante y la disposición al riesgo influyen de forma directa y significativa en la conducta intraemprendedora social de los estudiantes, explicándola en un $46,5 \%$, una gestión sistémica de éstas variables debe promover el intraemprendimiento social, generando un "ecosistema innovador", como se denomina al conjunto de elementos que se retroalimenta para ser sostenible, o sea, generar esquemas de colaboración entre distintos actores como empresas, universidades y gobierno, que al combinar sus esfuerzos crean un contexto que favorezca el surgimiento y sostenibilidad de nuevos productos y modelos de trabajo (de la Fuente et al., 2010). 
El valor moderado de explicación de la conducta intraemprendedora social deja al descubierto que se deben incorporar nuevas variables para lograr una mayor explicación del modelo, estas pueden ser: valencia de la conducta intraemprendedora social en la red interna del estudiante, tiempo disponible del estudiante, contribución en la empleabilidad, capacidad del estudiante para innovar, valores y actitud del estudiante para innovar y motivación, entre otras. Así entonces, un intraemprendedor en una IES, es el estudiante que se comporta como emprendedor dentro de la organización educativa, debido a que sus niveles de satisfacción, identificación y fidelidad lo motivan a implementar proyectos que vayan en beneficio de la IES, contribuyendo en la innovación de su cadena de valor y asumiendo riesgos asociados a la realización de actividades complementarias a las actividades formales del pregrado.

El Intraemprendimiento Social es la herramienta mediante la cual una empresa, no solo localiza las mejores mentes para encontrar ideas y soluciones, sino que integra a estas personas en el proceso de innovación, dejándolas convertir sus ideas en proyectos y ver como estos proyectos derivan en beneficios internos a la sociedad (Romero, 2012). En este contexto, la correlación entre innovación social y conducta intraemprendedora social representa un paradigma de gestión educacional que es prioritario en Chile, dado los actuales desafíos de la educación superior, donde es necesario que las organizaciones de educación integren de forma planificada al estudiante en la co-creación de innovaciones en procesos de apoyo y en los servicios educacionales. Las estrategias centradas en mejorar la innovación social en Universidad de La Serena, se deben convertir en decisiones estratégicas, pues son consistentes con su modelo educativo y con las competencias que crecientemente están demandando el mercado laboral y la sociedad. Por otra parte, la identificación incide positiva y directamente en la variable fidelidad del estudiante explicándolo en un $28 \%$, esta variable es un factor que aporta en explicar la conducta intraemprendedora social del estudiante, cabe recalcar que esto no significa que los estudiantes de la Facultad no sientan Fidelidad por su casa de estudios, sino que la Fidelidad si incide en la Conducta Intraemprendedora Social de los alumnos.

La gestión de la satisfacción del proceso enseñanza - aprendizaje y la satisfacción con la imagen Institucional, influirán directa y positivamente con significancia estadística, en la identificación del estudiante con su carrera. No tienen influencia estadística las otras dos variables que son parte del modelo: satisfacción del proceso de apoyo social-profesional y la satisfacción del proceso de apoyo interno. O sea, los estudiantes se comprometen con su carrera, si las decisiones de sus directivos hacen "rentable la inversión" que ellos realizan durante el pregrado, al proveer una educación de calidad y simultáneamente potencian la marca e imagen corporativa que proyecta una IES, variables que en su conjunto influyen en lograr una colocación laboral consistente con la carrera. Los resultados univariantes de esta investigación evidencian que no se gestionan sistémicamente las seis variables que influyen en la conducta intraemprendedora social, pues sus niveles se encuentran en un rango bajo a medio, en el caso de satisfacción proceso de enseñanza y aprendizaje, los resultados alcanzan un nivel de un 68,6\%; satisfacción de imagen institucional 62,6\%; identificación del estudiante un $65 \%$; fidelidad del estudiante un $69,5 \%$; innovación social un $63 \%$, y disposición al riesgo $56,4 \%$, generando en su conjunto bajos niveles de conducta intraemprendedora social en los estudiantes equivalente a un $57,2 \%$.

Junto a lo anterior, se destacan los resultados del análisis bivariante que permiten obtener la relación que existe entre el género, edad y carrera, con la Conducta Intraemprendedora Social. Bajo el contexto anterior, al realizar el test de Fisher, en todos los casos se rechaza la hipótesis alterna, pues el F calculado es menor al $\mathrm{F}$ tabular (al $5 \%$ de significancia), por lo tanto, el nivel de la conducta intraemprendedora social de los estudiante de la Facultad de Ingeniería, es independiente tanto del género, la edad y la carrera de los mismos, pues no se evidencian diferencias significativas estadísticamente. La universidad de La Serena y en general las IES deben establecer estrategias con el propósito de fortalecer ecosistemas que instalen una cultura de innovación de los estudiantes que lo lleven a liderar iniciativas extracurriculares mientras asume un rol activo en sus cursos de pregrado, lo que refleja su mayor disposición al riesgo, y que fortalezca la identificación con la misma Universidad. O sea, esto impactará positivamente en la calidad de los procesos de enseñanza aprendizaje, y de esta forma se incrementarán los índices de identificación y/o fidelidad, rol activo del estudiante, rol de maestro y facilitador del docente y en consecuencia en las conductas.

\section{CONCLUSIONES}

Se concluye que tanto la innovación social, la fidelidad del estudiante y la disposición al riesgo logran explicar a la conducta intraemprendedora social de los estudiantes de la Facultad de Ingeniería de la Universidad de La Serena. Mientras mayor sea la identificación de los estudiantes mayor será la fidelidad que éstos sientan por su casa de estudios, impactando positivamente en la conducta intraemprendedora social de los estudiantes.

La gestión de los directivos de una IES en cuanto a la satisfacción del proceso de apoyo social-profesional y la satisfacción del proceso de apoyo interno, no influyen con significancia estadística en la identificación del 
estudiante con su carrera, sin embargo ello no implica que se debe descartar su gestión operativa y estratégica, dado que proveen las "condiciones mínimas" esperadas en una IES, según las normas de acreditación institucional.

La satisfacción por la imagen institucional y la satisfacción derivada de los procesos de enseñanza aprendizaje son las únicas que logran incidir en la identificación de los estudiantes con su universidad, y dada su relevancia en aumentar el valor de la IES, a través de internalizar los beneficios de la conducta intraemprendedora social, instala en sus directivos el desafío de gestionar los stakeholders que son parte de su entorno, pues representan proveedores de recursos, capacidades y conocimientos que les permitirá crecientemente mejorar la identificación del estudiante y sus variables causales.

La gestión sistémica de las instituciones de educación superior se debe enfocar en fortalecer la innovación social, fortaleciendo la disposición al riesgo de los estudiantes y mejorar los procesos de enseñanza aprendizaje e imagen institucional, así se aporta en la formación de estudiantes con conductas intraemprendedoras y a iniciar el camino de construir IES de clase mundial.

Un estudiante al tener una Conducta Intraemprendedora Social tiene más posibilidades para potenciar y usar esas capacidades cuando estén laborando en las empresas públicas o privadas, o bien, generar emprendimientos de valor propio, contribuyendo así al logro del perfil de egreso, del modelo educativo de las IES y al desarrollo de su región y país.

\section{AGRADECIMIENTOS}

Los autores agradecen el apoyo entregado por la Universidad de La Serena para terminar exitosamente esta investigación.

\section{REFERENCIAS}

Annamdevula, S. y R. Bellamkonda, The effects of service quality on student loyalty: the mediating role of student satisfaction, Journal of Modelling in Management, 11(2), 446-462, (2016)

Bertoglia, L., La interacción profesor-alumno, una visión desde los procesos atribucionales, Psico Perspectivas, 4, 57-73, (2005)

Cabana, S. R., F. Cortés, D. Vega y R. Cortés, Análisis de la Fidelización del Estudiante de Ingeniería con su Centro de Educación Superior: Desafíos de Gestión Educacional, Formación Universitaria, 9(6), 93-104, https://doi.org/10.4067/S0718-50062016000600009, (2016)

Candelas, C., M. Gurruchaga, A. Mejías y L. Flores, Medición de la satisfacción estudiantil universitaria: Un estudio de caso en una institución Mexicana, Rev. Iberoamericana de Ingeniería Industrial, 5(9), 261-274, (2013)

Capriotti, P., Planificación Estratégica de la Imagen Corporativa, Editorial IIRP, Málaga, España, (2013)

Chin, W.W., The Partial Least Squares Approach to Structural Equation Modeling, in G.A. Marcoulides [Ed.], 295-336, Modern Methods for Business Research, Mahwah, NJ: Lawrence Erlbaum Associates, (1998)

Cokley, K., M. Komarraju, N. Patel, J. Castillon, R. Rosales, R. Pickett, S. Piedrahita, J. Ravitch y L. Pang, Construction and initial validation of the student-professor interaction scale, The college of student affairs journal, 24(1), 32-50, (2004)

de la Fuente, H., M. Marzo y M. Reyes, Análisis de la satisfacción universitaria en la facultad de ingeniería de la universidad de Talca, Ingeniare. Revista chilena de ingeniería, 18(3), 350-363, (2010)

Donoso, S. y E. Schiefelbein, Análisis de los modelos explicativos de retención de estudiantes en la universidad: una visión desde la desigualdad social, Estudios Pedagógicos, 33(1), 7-27, (2007)

Esen, M. y R. Sekerdil, The Effects Of Intrapreneurship on Social Innovation: A Research in Izmir, International Journal of Contemporary Economics and Administrative Sciences, 7(1-2), 13-30, (2017)

Esposito, V., W. Chin, J. Henseler y H. Wang, Handbook of Partial Least Squares: Concepts, Methods and Applications, 1st edition, Berlin: Springer-Verlag Berlin Heidelberg, (2010)

Falk, R. y N. Miller, A primer for soft modeling, 1ra edition, Akron, O H: University of Akron Press, (1992)

Forcael, E., S., Vargas, A., Opazo y L., Medina, Rol del ingeniero civil en la sociedad chilena contemporánea, Revista de la Construcción, 12(2), 72-87, (2013)

Fullerton, C., Creating advocates: The roles of satisfaction, trust and commitment, Journal of Retailing and Consumer Services, 18(1), 92-100, (2011)

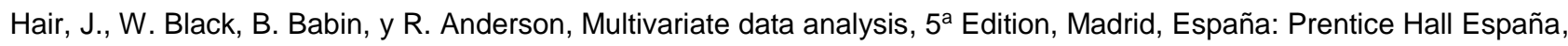
(2009)

Hair, J., C. Ringle y M. Sarstedt, PLS-SEM: Indeed a Silver Bullet, Journal of Marketing Theory and Practice, 19(29), 139151, (2011) 
Helgesen, $\varnothing$. y E. Nesset, What accounts for students' loyalty? Some field study Evidence, International Journal of Educational Management, 21 (2), 126-143, (2007)

Klem, A. y J. Connell, Relationships matter: Linking teacher support to student engagement and achievement, The Journal of School Health, 74(7), 262-273, (2004)

Kumar, M. y H. Jauhari, Satisfaction of learning, performance, and relatedness needs at work and employees' organizational identification, International Journal of Productivity and Performance Management, 65(6), 760-772, (2016)

Kwong, K., Partial Least Squares Structural Equation Modeling (PLS-SEM) Techniques Using SmartPLS, Marketing Bulletin, 24, 1-32, (2013)

Lozano, J., R. Hernández, J. Díaz y A. Fernández, Intraemprendimiento social Universitario. Estudio de casos, Revista Investigación Multidisciplinaria, Edición Especial de Emprendimiento, 21-45, (2015)

Martínez, Z., M.F. Páramo, M.A. Guisande, C. Tinajero, L. da Silva y M.S. Rodríguez, Apoyo social en universitarios españoles de primer año: propiedades psicométricas de Social Support Questionnarie-Short Form y el Social Provisions Scale, Revista Latinoamericana de Psicología, 46(2), 102-110, (2014)

McNeely, C. y C. Falci, School connectedness and the transition into and out of health - risk behavior among adolescents: A comparison of social belonging and teacher support, Journal of School Health, 74(7), 284 - 292, (2004)

Méndez C. y M. Rondón, Introducción al análisis factorial exploratorio, Revista Colombiana de Psiquiatría, 41(1), 197-207, (2012)

Moriano J., G. Topa, E. Valero y J. Levy, Identificación organizacional y conducta intraemprendedora, Anales de Psicología, 25(2), 277-287, (2009)

Moriano J., F. Molero, G. Topa y J. Levy, The influence of transformational leadership and organizational identification on intrapreneurship, International Entrepreneurship and Management Journal, 10(1), 103-119, (2014)

Nijhof, A., J.C. Looise, J., de Leede, Social Intrapreneurship: A Conceptual, Theoretical And Empirical Exploration of its Meaning and Contribution, in Innovation, Social Responsibility, Creativity, Ethics and Olaf Fisscher by P. de Weerd, K. Visscher y J. Visser, pp. 109-128, Enschede, Países Bajos, (2012)

Pereira, M., Nuevas tendencias en la evaluación de la calidad de las universidades: los índices de calidad percibida y satisfacción de los egresados (con modelos de ecuaciones estructurales), 39(3), 73-84, (2011)

Petrella, C., Gestión de la relación de las Universidades con Docentes, Estudiantes y Egresados, Revista lberoamericana de Educación, ISSN: 1681-5653, 47(5), (2008)

Pratt, M., To be or not be?: Central questions in organizational identification, in Identity in Organizations: Building Theory Through Conversations by D.A Whetten, y P.P Godfey, pp. 171-208, California, E.E.U.U, (1998)

Quintero, C. Generación de competencias en jóvenes emprendedores. UNAB, https://goo.gl/W9jonN, (2007)

Romero, G., Intraemprendimiento, la innovación dentro de las organizaciones, https://goo.gl/etj6ug, (2012)

Salcedo, J., La importancia de los intraemprendedores en las empresas, goo.gl/n9U6Um, (2012)

Schlesinger, W., A. Cervera y H. Calderón, El papel de la confianza, la imagen y los valores compartidos en la creación de valor y lealtad: aplicación a la relación egresado-universidad, Revista Española de Investigación de Marketing ESIC, 18, 126-139, (2014)

Scott, S. y L. Lane, A Stakeholder Approach to Organizational Identity, The Academy of Management Rewiew, 25(1), 4362, (2000)

Sung, M. y S. Yang, Toward the Model of University Image: The Influence of Brand Personality, External Prestige, and Reputation, Journal of Public Relations Research, 20(4), 357-376, (2008)

Tenenhaus M., V., Esposito, Y. Chatelin y C., Lauro, PLS path modeling, Computational Statistics \& Data Analysis, 48(1), 159-205, (2005)

Topa, G., J. Moriano, J. Morales y A. Moreno, Identificaciones múltiples y ciudadanía en el trabajo: mediación de las fuentes de apoyo social, Revista Latinoamericana de Psicología, 42(3), 427-436, (2010)

Villa, A., La innovación Social en el Ámbito Universitario: Una Propuesta Para su Diagnóstico, Revista Argentina de Educación Superior, (8), 188-218, (2014)

Willie, C., Confidence, trust and respect: The preeminent goals of educational reform, The Journal of Negro Education, 69(4), 255-262, (2000)

Yáñez, R., V. Pérez y C. Yáñez, La confiabilidad hacia los docentes por parte de los estudiantes y su influencia en la construcción de confianza y en la identificación organizacional hacia sus carreras, Estudios Pedagógicos XXXI, 3(2), 89103, (2005) 